\title{
A Topological Model of the Solar Magnetic Field Reversals
}

\author{
E.E. Benevolenskaya \\ Pulkovo Astronomical Observatory \\ 196140, Leningrad, USSR
}

The phenomenon of a three-fold reversal of the solar polar magnetic field in both hemispheres has not been observed during the last 115 years. Such three-fold reversals took place in the southern hemisphere alone in the even cycles Nos 12 (1885.8), 14 (1908.4) and in the northern hemisphere alone in solar cycles Nos 16 (1928.5), 18 (1949.0), 20 (1970.6). The single reversal took place in the odd cycles, the only exception is the solar cycle No 19 (Fig. 1).

\begin{tabular}{|c|c|c|c|c|c|c|}
\hline CYCLES & $10-11$ & $12-19$ & $14-18$ & $10-17$ & $10-10$ & $20-21$ \\
\hline $\mathbf{N}$ & $+1-$ & $-1+\quad+1-$ & $-1+\quad+1-$ & $-1+1-1++1-$ & $-1+1-1++1-1+1-$ & $-1+1-1++1-$ \\
\hline$s$ & $-1+$ & $+1-1+1--1+$ & $+1-1+1--1+$ & $+1-\quad-1+$ & $+1-\quad-1+$ & $+1-\quad-1+$ \\
\hline
\end{tabular}

Fig. 1. Hale's 22-year cycles and the reversal of the Sun's magnetic fields in the odd and even 11-year cycles in the northern $(N)$ and southern $(S)$ hemispheres.

There are periods of 1.7-2.5 years in the variation of background magnetic fields (Makarov et al., 1985). It determines the quasi-period of the high-frequency component and corresponds to a time interval between the zones of alternating polarity of the magnetic field. This enables us to show topologically that single and three-fold polarity reversals of the solar magnetic fields can result from interaction of two types of magnetic fields: a low-frequency component with period of the order of 20 years and a high frequency component with period of order of 1.7-2.5 years (Benevolenskaya and Makarov, 1990).

According to modern concepts, solar magnetic fields are generated by helicity and the differential rotation of the solar convection zone (Parker, 1979). The dynamo process in the linear approximation is instrumental in generating one carrier 
frequency $\omega_{\mathrm{c}}$. Within the frame of linear stochastic theory, a spectrum of frequencies is generated for the dynamo numbers $D>D_{\mathrm{c}}$. In the non-linear theory, stable frequencies with a period shorter than that of a cycle may occur (Hoyng, 1990). The toroidal fields $\left(B_{\varphi}\right.$-component) formed in the convection zone show up as sunspots on the surface. The background magnetic fields ( $B_{\mathrm{r}}$-component) in their turn are transferred poleward due to diffusion and meridional circulation (Wang et al., 1989). Let us assume that

$$
B_{\mathrm{r}}=B_{\mathrm{r}}^{\prime}+\delta B_{\mathrm{r}},
$$

where $B_{\mathrm{r}}^{\prime}$ is a low-frequency component with a period of the order of 22 years and $\delta B_{\mathrm{r}}$-is a high-frequency component.

According to Makarov et al. (1987) and Kleeorin and Ruzmaikin (1988) the $B_{\mathrm{r}}$ field can be represented as a dynamo wave

$$
B_{\mathrm{r}}^{\prime}=b_{\mathrm{r}}(r, \theta) \cos \left(\omega_{\mathrm{c}} t+g(r, \theta)-\frac{\pi}{2}\right),
$$

where $b_{\mathbf{r}}(r, \theta)$ is the amplitude of the radial field in the convection zone; $g(r, \theta)$ is the phase function, $g\left(R_{\odot}, 0\right)=0 ; \omega_{\mathrm{c}}=\frac{2 \pi}{T_{\mathrm{c}}}$ is the frequency of the solar cycle. Similarly, the $\delta B_{\mathrm{r}}$ field will be represented as

$$
\delta B_{\mathrm{r}}=\delta b_{\mathrm{r}}(r, \theta) \cos \left(\omega_{\delta}(t) t+g_{1}(r, \theta)-\frac{\pi}{2}\right) .
$$

If $\omega_{\delta}(t)$ is a slowly varying with time and $\omega_{\delta}(t)=\omega_{\mathrm{c}}=$ const and $g_{1}(r, \theta)=g(r, \theta)+\varphi$, $b_{\mathrm{r}}(r, \theta)=A \cdot \delta B_{\mathrm{r}}(r, \theta)$. At some fixed latitude $\theta_{\mathrm{c}}$ :

$$
B_{\mathrm{r}}=b_{\mathrm{r}}\left(r, \theta_{\mathrm{c}}\right) \sin \omega_{\mathrm{c}} t+\delta b_{\mathrm{r}}\left(r, \theta_{\mathrm{c}}\right) \sin \left(\omega_{\delta} t+\varphi\right) .
$$

Consider the domain $B_{\mathrm{r}}=0$. The function

$$
B_{\mathrm{r}}=b_{\mathrm{r}}(r, \theta) \sin \omega_{\mathrm{c}} t+\frac{b_{\mathrm{r}}(r, \theta)}{A} \sin \left(\omega_{6} t+\varphi\right)
$$

becomes zero depending on the relation between the phase $\varphi$, frequencies $\omega$ and $\omega_{\delta}$, and the relation of the amplitudes $A$. If the frequencies are multiple, i.e. $\omega_{\delta}=K \omega_{\mathrm{c}}$ and $K$ integer, e. g. $K=14, A=2.5$. This corresponds to 18 months for real $T_{\mathrm{c}}=20.6$ years. For $K=8$ and $A=2.5$ the behaviour of the $B_{\mathrm{r}}^{\prime}$ field (solid line) is shown in Fig. 2a, the $\delta B_{\mathrm{r}}$ field in Fig. $2 \mathrm{~b}$ and $B_{\mathrm{r}}$ in Fig. 2c. The polarity distribution of the $B_{\mathrm{r}}$ field on the solar surface given in Fig. 2d.

When the polarity of the background field low-frequency component reverses from - to + the zones of alternating polarities are formed. Such a situation takes place in the northern hemisphere, beginning with solar cycle No 16 . The presence of such zones depend on the relationship of amplitudes and phases of the highfrequency and low-frequency component of the background magnetic field. The combination of the phase and growth of the amplitude $\delta B_{\mathrm{r}}$ can result in a situation in which such zones form with the reversal of the $B_{\mathrm{r}}^{\prime}$ field from + to - , which actually took place in solar cycle 19. The width of the zones of the alternating 
polarities approximately corresponds to the period of the field (Fig. 2). It is of the order of $1.5-2.0$ years.

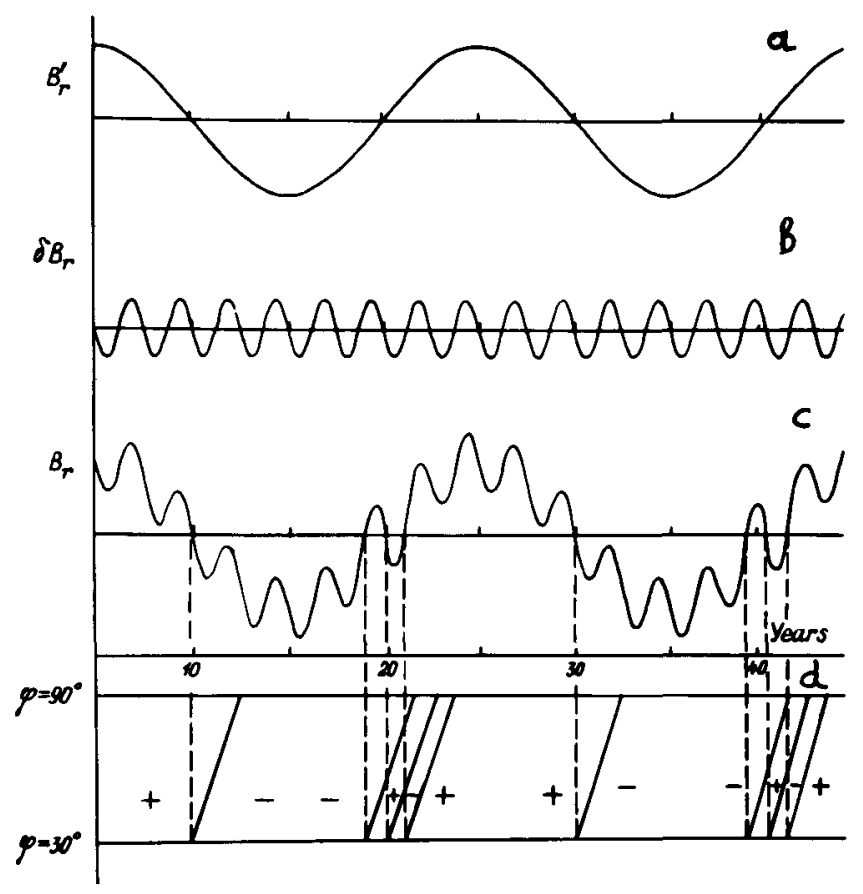

Fig. 2. Schematic representation of the behaviour of $B_{\mathrm{r}}^{\prime}, \delta B_{\mathrm{r}}$ and $B_{\mathrm{r}}$.

Thus, three-fold reversals of the high latitude magnetic field of the Sun can be interpreted as a result of an interaction of the low-frequency component of the field, describing the behaviour of mean background fields during the 22-year period, with a high-frequency component with a slowly-varying phase.

\section{References}

Benevolenskaya, E.E. and Makarov, V.I.: 1990, Soln. Dann. No 5, 75

Hoyng, P.: 1990 in Solar Photosphere: Structure, Convection and Magnetc Fields, ed.

Stenflo, O.J., IAU Symp. No 138, p. 359

Kleeorin, N.I. and Ruzmaikin, A.A: 1988, Preprint IZMIRAN, No 7(833), p. 3

Makarov, V.I., Tavastsherna, K.S. and Petrova, N.S.: 1985, Soln. Dann. No 6, 69

Parker, E.N.: 1979, Cosmical Magnetic Fields, Oxford University Press.

Wang, J.W., Nash, A.G. and Sheeley, N.R.,Jr.: 1989, Astrophys. J. 347, 529 